University of Wollongong

Research Online

Faculty of Engineering - Papers (Archive)

Faculty of Engineering and Information

Sciences

June 2005

\title{
The effect of the electron energy spectrum on electronic efficiency and power in thermionic and thermoelectric devices
}

\author{
Mark F. O'Dwyer \\ University of Wollongong, markod@uow.edu.au \\ T. E. Humphrey \\ University of California Santa Cruz, USA \\ R. A. Lewis \\ University of Wollongong, roger@uow.edu.au \\ C. Zhang \\ University of Wollongong, czhang@uow.edu.au
}

Follow this and additional works at: https://ro.uow.edu.au/engpapers

Part of the Engineering Commons

https://ro.uow.edu.au/engpapers/73

\section{Recommended Citation}

O'Dwyer, Mark F.; Humphrey, T. E.; Lewis, R. A.; and Zhang, C.: The effect of the electron energy spectrum on electronic efficiency and power in thermionic and thermoelectric devices 2005.

https://ro.uow.edu.au/engpapers/73

Research Online is the open access institutional repository for the University of Wollongong. For further information contact the UOW Library: research-pubs@uow.edu.au 


\title{
The Effect of the Electron Energy Spectrum on Electronic Efficiency and Power in Thermionic and Thermoelectric Devices
}

\author{
M. F. O'Dwyer ${ }^{1}$, T. E. Humphrey ${ }^{2}$, R. A. Lewis ${ }^{1}$ and C. Zhang ${ }^{1}$ \\ ${ }^{1}$ School of Engineering Physics and Institute for Superconducting and Electronic Materials \\ University of Wollongong, Wollongong NSW 2522, Australia \\ ${ }^{2}$ Baskin School of Engineering, University of California Santa Cruz, Santa Cruz, CA 95064-1077, USA
}

\begin{abstract}
We show that the details of the energy spectrum of transmitted electrons in thermionic and thermoelectric devices have a significant impact on their performance. We distinguish between traditional thermionic devices where electron momentum is filtered in the direction of transport only and a second type, in which the electron filtering occurs according to total electron momentum. Our main result is that the electronic efficiency of a device is not only improved by reducing the width of the transmission filter, but also strongly depends on whether the transmission probability rises sharply from zero to full transmission. Finally, we comment on the implications of the effect the shape of the electron energy spectrum has on the efficiency of thermoelectric devices and suggest an experimental measure for providing insight into the nature of the electron energy spectrum.
\end{abstract}

\section{Introduction}

Recent advances in solid-state nanostructure design offer the possibility of engineering the energy spectrum of electrons transmitted through the device in a way that was not possible in traditional vacuum thermionics or bulk thermoelectrics. In light of the new design freedom offered by nanostructuring, it is useful to re-examine the impact of the electron energy spectrum upon what Hatsopoulos has called the 'electronic efficiency' of thermionic devices, defined as the efficiency associated with strictly electronic processes under ideal conditions of particle transport [1]. Improvements in electronic efficiency due to better device design which can be achieved without lowering the power will translate into an improvement in the operating efficiency of practical thermionic devices where non-ideal effects are important.

In this paper, we analyze in detail the dependence of the electronic efficiency of thermionic power generators and refrigerators upon the details of the energy spectrum of electrons transmitted ballistically between the emitter and collector. We examine two idealized models of thermionic nanodevices. In the first, energy (or more precisely, momentum) filtering of electrons occurs in the direction of transport only. This model, which we will denote as a ' $k_{x}$ filtered thermionic device', is applicable to single barrier or multibarrier (superlattice) solid-state devices [2,3]. In the second model, which we will denote as a ' $k_{r}$ filtered thermionic device', energy filtering of the total energy of electrons is assumed to be possible. This model is applicable to devices where there is non-conservation of lateral momentum [4] and quantum dot superlattices or superlattice nanowires. We also compare the dependancies of thermionic and thermoelectric device efficiency on the details of the electron energy spectrum. Fig. 1 shows geometrically the range of electrons transmitted in idealized $k_{x}$ and $k_{r}$ devices in momentum space.

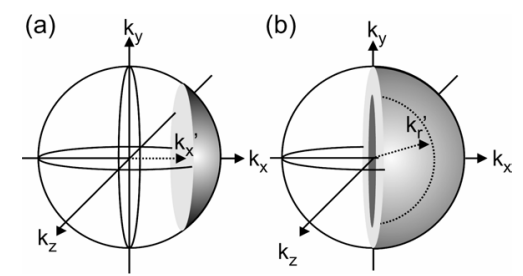

Figure 1: Fermi spheres indicating electrons transmitted by (a) a $k_{x}$ filtered device and (b) a $k_{r}$ filtered device.

\section{Transport Theory}

The net electrical current density flowing from the cold to hot reservoir is given by [5]

$$
J_{r / x}=q \int_{0}^{\infty}\left[n_{r / x}^{C}-n_{r / x}^{H}\right] \zeta\left(E_{r / x}\right) d E_{r / x}
$$

for a $k_{r} / k_{x}$ device where $q=-1.602 \times 10^{-19} \mathrm{C}$ is the charge of an electron, $\zeta$ is the transmission probability, $E_{r / x}$ is the total energy/energy in the $x$ direction,

$$
n_{r}^{C}=\frac{m^{*} E}{2 \pi^{2} \hbar^{3}}\left[1+\exp \left(\left(E_{r}-\mu_{C / H}\right) / k_{B} T_{C / H}\right)\right]^{-1}
$$

and

$$
n_{x}^{C}=\frac{m^{*} k_{B} T_{C / H}}{2 \pi^{2} \hbar^{3}} \log \left[1+\exp \left(-\left(E_{x}-\mu_{C / H}\right) / k_{B} T_{C / H}\right)\right]
$$

are the number of electrons with total $/ x$ energy, $E_{r / x}$, arriving at the reservoir interface per unit area per unit time and $\mu_{\mathrm{C} / \mathrm{H}}$ and $T_{C / H}$ are the chemical potentials and temperatures respectively of the cold and hot reservoirs.

One may calculate the heat flux out of the hot and cold reservoirs by noting that an electron leaving or entering the cold/hot reservoir will remove or add respectively an amount of heat equal to the difference between the total energy of the electron and the Fermi energy of the reservoir, that is $E-\mu_{C / H}$ where $E$ is the total electron energy. For a $k_{r}$ device, $E=E_{r}$. The total average energy of an electron leaving the cold/hot reservoir in a $k_{x}$ device is $E=E_{x}+k_{B} T_{C / H}$. The resultant heat current out of the cold/hot reservoir is [5]

$$
\dot{Q}_{r / x}^{C / H}=\mp \int_{0}^{\infty}\left(E-\mu_{C / H}\right)\left[n_{r / x}^{C}-n_{r / x}^{H}\right] \xi\left(E_{r / x}\right) d E_{r / x} .
$$

Thermoelectric devices are generally differentiated thermionic devices according to whether electron transport is ballistic or diffusive [2]. There is, however, little to distinguish the underlying thermodynamics of the two types of devices. It may be shown that the diffusive and ballistic formalisms reduce to the same form for devices with length around the electron mean free path implying a similar dependence on the properties of the electron energy spectrum [6]. 
The electronic efficiency of a thermionic device or the intensive efficiency across a small section of thermoelectric material acting as a power generator is given by

$$
\eta_{P G}=V J / \dot{Q}
$$

and the electronic/intensive COP when the system is acting as a refrigerator is given by

$$
\eta_{P G}=\dot{Q} / V J
$$

\section{Effect of Transmission Probability Width}

To achieve reversibility in a thermionic or thermoelectric device, electrons must flow at energies where the Fermi occupation of states is constant $[7,8]$. One way to achieve this, which we refer to as energy-specific equilibrium, is to transmit electrons at only a single energy where the Fermi occupation throughout the device is equal, $E_{0}=[\mu(T+\delta T)$ $(\mu-\delta \mu) T] / \delta T[5,7]$, where $\delta T$ and $\delta \mu$ are the temperature and chemical potential changes respectively over a $\delta x$ in the device. This may be achieved in a ballistic device by transmitting electrons at $E_{0}$ only. In a diffusive device inhomogeneous doping or a graded band structure is required with a delta function DOS [7]. Unlike a $k_{r}$ thermionic device, a $k_{x}$ thermionic device is limited to efficiency below the Carnot value due to the fact the two unfiltered degrees of freedom introduce a spread in the total energy, as shown in Fig. 2.

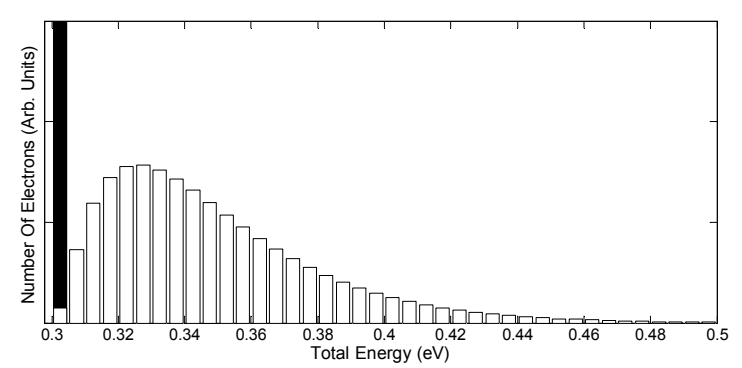

Figure 2: The total energy distributions for electrons leaving a reservoir in $k_{x}$ (white) and $k_{r}$ (black) filtered thermionic devices with a filter of $5 \mathrm{meV}$ at $0.3 \mathrm{eV}$. The vertical axis has been cut off for clarity of the details in the $k_{x}$ system values. $T$ $=270 \mathrm{~K}$ and $\mu=0.1 \mathrm{eV}$.

We now analyze the effect of filter width, with a rectangular filter of finite width, where all electrons are transmitted in the range $E_{r / x}{ }^{\prime}<E_{r / x}<E_{r / x}{ }^{\prime}+\Delta E_{r / x}$. The results for filters of width $0.01 k_{B} T_{C}$ to $100 k_{B} T_{C}$, with $E^{\prime}$ tuned for maximum efficiency, are shown in Fig. 3. The $k_{r}$ and $k_{x}$ systems may achieve an efficiency/COP approximately equal to the maximum value for filter widths of about $0.1 k_{B} T_{C}$ and $k_{B} T_{C}$ respectively, which are achievable with practical devices. As the filter widths progress beyond these values the efficiency/COP drops and then plateaus again at a final value. Large filter widths effectively correspond to the situation where all electrons above $E^{\prime}$ are being transmitted. As the distribution function rapidly converges to zero at high energies, this means that further increasing filter width has a minimal effect upon the electronic efficiency. The $k_{r}$ device has higher electronic efficiency than the $k_{x}$ device due to a greater concentration of electrons around the energy-specific equilibrium energy $E_{0}$.

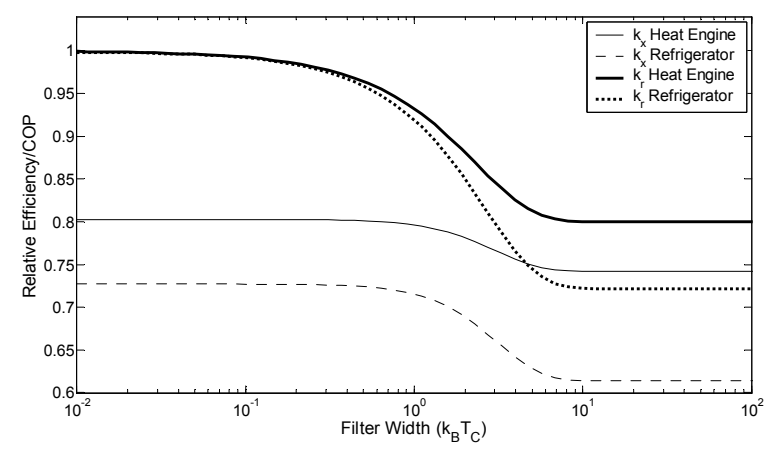

Figure 3: The maximum relative efficiency and COP as a fraction of the Carnot limit for $k_{r}$ and $k_{x}$ filtered thermionic devices versus the width of the filter from $0.01 k_{B} T_{C}$ to $100 k_{B} T_{C} . T_{H}=300 \mathrm{~K}, T_{C}=270 \mathrm{~K}, \mu_{H}=0.98 \mathrm{eV}$ and $\mu_{C}=1.00$ $\mathrm{eV}$.

\section{Effect of Transmission Probability Slope}

We now consider the effect upon the electronic efficiency of a gradual transition, which more closely resembles the shape of the transmission probability in practical devices. We begin by using two convenient 'artificial' transmission probabilities, the slope of which can be easily varied. The first, a Gaussian peak which might approximate the transmission probability of a resonance, is given by $\zeta\left(E_{r / x}\right)=$ $\exp \left[-\left(E_{r / x}-E_{c}\right)^{2} / w\right]$ where $E_{c}$ defines the center energy of the peak and $w$ is a width parameter which is used to vary the sharpness of the slope. The second artificial transmission probability considered is a 'half-Gaussian', intended to approximate the transmission probability of a single barrier of finite width. This is given by the Gaussian equation for $E_{r / x} \leq$ $E_{C}$ and is equal to one for $E_{r / x}>E_{C}$. The sharpness of the Gaussian and half-Gaussian transmission probabilities were varied between $w=10^{-5}$, corresponding to an ideal filter or perfectly sharp single barrier transmission probability, and $\mathrm{w}$ $=0.006$. Gaussian and half-Gaussian transmission probabilities are shown in Fig. 4.
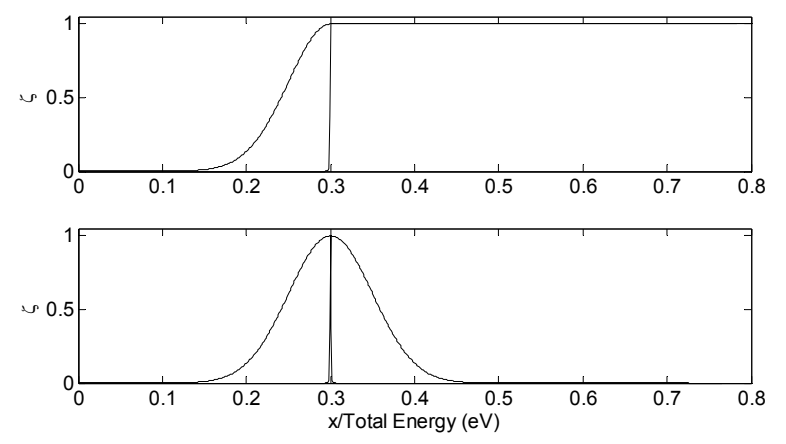

Figure 4: Artificial transmission probabilities generated using a Gaussian function (top) and a half-Gaussian function (bottom). The two extremes of width parameter are shown, $\mathrm{w}$ $=0.1$ (slowly rising) and $\mathrm{w}=0.00001$ (sharply rising).

Fig. 5 shows the maximum COPs/efficiencies associated with room temperature refrigerators and heat engines operating at higher temperatures. Since all electrons of energy 
other than $E_{0}$ reduce the electronic efficiency, we expect the sharpest peak in Fig. 5 to yield the highest efficiency/COP and this is confirmed by the numerical results. The most interesting result, however, is that the electronic efficiency of the half-Gaussian transmission probability is very strongly dependent upon how sharply the transmission rises from zero to unity. A smooth rise in the transmission probability lowers the electronic efficiency for the same physical reason that a $k_{x}$ filtered device has a lower electronic efficiency than a $k_{r}$ filtered device, that is, the spread of electrons energies away from $E_{0}$. This will have implications on device design as will be discussed in the next section. A perfectly sharp transmission probability is also desirable for high power and it may be shown that maximum heat engine power is realized with a transmission probability which is equal to one for all energies greater than $E_{0}[9]$.
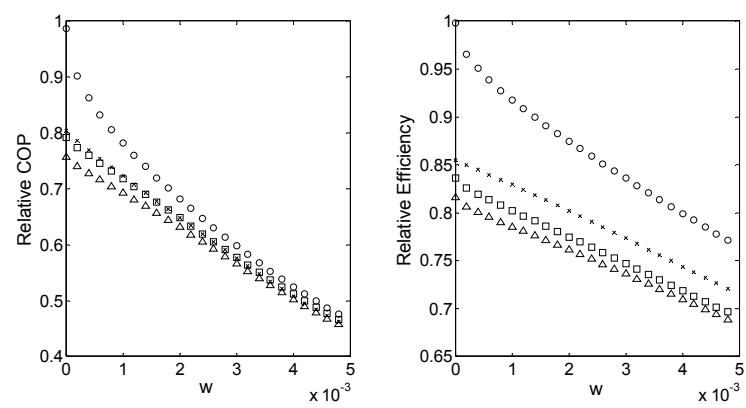

Figure 5: The efficiency/COP relative to the Carnot value of a $k_{r}$ Gaussian (circle), $k_{x}$ Gaussian (cross), $k_{r}$ half-Gaussian (square) and $k_{x}$ half-Gaussian (triangle) for refrigerator systems (left) $\left(T_{H}=300 \mathrm{~K}\right.$ and $\left.T_{C}=270 \mathrm{~K}\right)$ and heat engine systems (right) $\left(T_{H}=900 \mathrm{~K}\right.$ and $\left.T_{C}=300 \mathrm{~K}\right)$ versus width parameter with $\mu_{C}=0.1 \mathrm{eV}$.

\section{The Electron Energy Spectrum and Practical Devices}

Semiconductor based devices, including superlattices, may be specifically designed to achieve desired energy spectrum features in $k_{x}$ devices. Narrow energy filters may be achieved through resonant tunneling, minibands, quantum dot structures, and potentially by emission from nanotubes and normal-insulating-superconductor junction (NIS) devices. It is likely, however, that the best way to simultaneously achieve high electronic efficiency and high power in a ballistic device is to design the structure such that the transmission probability rises sharply from zero to one and remains close to unity beyond this. Whilst the most obvious way to achieve a transmission probability of this nature is to utilize a single barrier with a width as large as possible, but less than the mean free path of electrons, an array of thin barriers can also be used to engineer a transmission probability that rises sharply from zero to unity in instances where wider barriers are undesirable. Fig. 6 shows the transmission probabilities for a narrow single barrier, which yields a very slowly rising transmission probability and low electronic efficiency, as well transmission probabilities associated with a wide single barrier and multibarrier system, both of which give high electronic efficiency.

As was discussed earlier, the energy spectrum in a diffusive device is determined by the product of the local DOS, the velocity squared and the relaxation time. In a pure ballistic device, since the DOS and velocity are fixed by the reservoirs, a sharp electron energy spectrum is achieved via a sharp transmission probability. In diffusive devices, both the DOS and electron velocity may change as the device structure changes. It may be a difficult optimization problem to design a structure where their product changes sharply from its minimum to maximum value as a function of energy and if one seeks to increase the DOS or its sharpness, care must be taken that this does not result in decreased current due to reduced velocity [10].
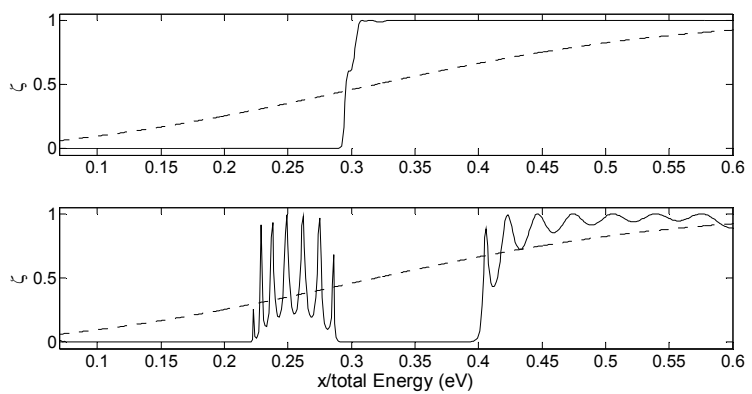

Figure 6: The transmission probabilities for a 5-nm single barrier (dashed) and 80-nm wide single barrier (solid, top) and 8 barrier device with 5 -nm barriers and wells (solid, bottom). $m^{*}=0.067 m_{e}$.

Mahan and Sofo have shown that the ideal transport distribution function for a thermoelectric device may be achieved with a delta function DOS [11]. Humphrey and Linke showed that the Carnot efficiency may be achieved in thermoelectric devices utilizing a delta function DOS and a graded device structure or inhomogeneous doping [7]. Their results are analogous to the results presented earlier in this paper where it was shown that the ideal transmission probability for a ballistic device was one which allowed the transmission of only a very narrow energy range of electrons. The results presented in this paper suggest that not only is the width of the energy spectrum important, but also whether it rises rapidly from zero to its maximum value. In practical devices, where loss mechanisms such as phonon heat leaks need to be considered, the magnitude of the energy spectrum also becomes important to the efficiency as the conductivity is given by the integral of the energy spectrum and occupation of states. Hicks and Dresselhaus have pointed out that the magnitude of the DOS can be increased by using structures of lower dimensionality, potentially increasing the power factor [12]. We also note that the DOS is also sharper for lower dimensional systems compared to bulk materials, which may result in an improved energy spectrum

\section{Experimental Properties Related to the Transmission Probability}

The presence of phonon heat leaks and contact resistance in solid-state thermionic devices makes direct measurements of the electronic efficiency difficult. Here we discuss an experimentally measurable property which may provide an indication of the shape of the electron energy spectrum and through this, electronic efficiency.

The I-V characteristics of a thermionic device are dependent on the voltage across the barrier system, $V_{B}$. As the 
bias is increased, the net electrical current decreases and reaches zero at the open circuit voltage $V^{B}{ }_{O C}$, from which the effective Seebeck coefficient, $S$, and energy-specific equilibrium energy may be calculated as

$$
E_{0}^{V_{O C}}=\mu_{C}+V_{O C}^{B} \frac{T_{C}}{T_{H}-T_{C}}=\mu_{C}+S T_{C} .
$$

$E_{0}^{V_{O C}}$ is the energy where the net number of electrons flowing from the hot to cold reservoir above it is equal to the net number flowing from the cold to hot reservoir below it. If we have a system with a transmission probability which is not perfectly sharp and electrons with energies lower than the barrier energy are transmitted (without significant change to the high energy details), for example through decreasing the barrier width, $E_{0}^{V_{O C}}$ is shifted to lower energy. Measuring this relative to a convenient energy, say the barrier energy or 'turn on' energy, provides a convenient sharpness indication for the transmission probability,

$$
\psi=S T_{C}-\varphi
$$

where $\varphi$ is the difference between the barrier or turn on energy and the chemical potential. A higher sharpness indicator is desirable, indicating a sharper transmission probability and therefore higher expected electronic efficiency and COP for power generation and refrigeration respectively. The sharpness indicator has the advantage over the Seebeck coefficient of being less dependent on the chemical potential/barrier height and more on the sharpness of the energy spectrum, as shown in Fig. 7. Here, the chemical potentials for a number of single barrier transmission probabilities have been varied to give a constant Seebeck coefficient as the barrier width and transmission probability sharpness changes. Fig. 7 shows the electronic efficiency varies significantly in this example. Whilst the Seebeck coefficient remains constant, the sharpness indicator increases as the electronic efficiency increases.

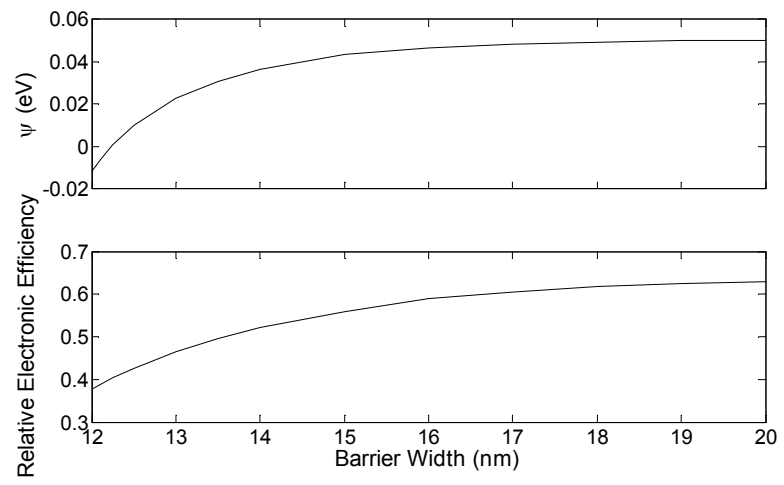

Figure 7: The maximum relative electronic efficiency (top) and sharpness indicator (bottom) versus single barrier width. The cold reservoir chemical potential has been tuned so that the Seebeck coefficient is constant for each barrier width. $T_{C}$ $=270 \mathrm{~K}, T_{H}=300 \mathrm{~K}$, barrier height of $0.3 \mathrm{eV}$ and effective mass of $0.067 m_{e}$.

\section{Conclusions}

We have shown that the nature of the electron energy spectrum has a significant impact on the performance of thermionic and thermoelectric devices. Electron efficiency in ballistic devices may be increased by transmitting a narrower range of electrons or by increasing the sharpness of the rise in the electron energy spectrum, which can also increase maximum power. Sharp electron energy spectra may be achieved with wide single barriers or carefully arranged multiple barriers. Due to the similarities between the diffusive and ballistic formalisms we expect thermoelectric devices to have the same dependence on the details of the electron energy spectrum for mean free path length devices. Finally, an experimental measure was suggested which can provide insight into the nature of the electron energy spectrum.

\section{Acknowledgments}

MO'D is supported by the Australian Research Council. $\mathrm{TH}$ is supported by the Australian Research Council and funding from ONR MURI. The authors acknowledge helpful discussions with Ali Shakouri and Heiner Linke.

\section{References}

1. Hatsopoulos, G. N. and E. P. Syftopoulos, Thermionic Energy Conversion Volume I: Processes and Devices, The MIT Press (London, 1973).

2. Mahan, G. D., Sofo, J. O., Bartkowiak, M., "Multilayer thermionic refrigerator and generator", J. Appl. Phys., Vol. 83, pp. 4683-4689 (1998).

3. Shakouri, A., LaBounty, C., Piprek, J., Abraham, P., Bowers, J. E., "Thermionic emission cooling in single barrier hetrostructures", Appl. Phys. Lett., Vol.74 pp. 8889 (1999).

4. Vashaee, D. and Shakouri, A., "Electronic and thermoelectric transport in semiconductor and metallic superlattices", J. Appl. Phys., Vol. 95, pp. 1233-1245 (2004).

5. O'Dwyer, M. F., Humphrey, T. E., Lewis, R. A. and Zhang, C., "Efficiency in nanostructured thermionic and thermoelectric devices", submitted to Phys. Rev. B.

6. Humphrey, T. E., O’Dwyer, M. F., Zhang, C. and Lewis, R. A., "Solid-state thermionics and thermoelectrics in the ballistic transport regime", J. Appl. Phys., in press.

7. Humphery, T. E. and Linke, H., "Reversible Thermoelectric Nanomaterials" Phys. Rev. Lett., Vol. 94, pp. 096601-1-4 (2005).

8. Humphrey, T. E., Newbury, R., Taylor, R.P. and Linke, H., "Reversible Quantum Brownian Heat Engines for Electrons", Phys. Rev. Lett., Vol. 89, pp. 116801-1-4 (2002).

9. Humphrey, T. E., O’Dwyer, M. F. and Linke, H., "Power Optimization in Thermionic Devices", J. Phys. D, in press.

10. Ali Shakouri, personal corresponence (2005).

11. Mahan, G. D. and Sofo, J. O., "The best thermoelectric", Nat. Academy of Sci., Vol. 93, pp. $7436-7439$ (1996).

12. Hicks, L. D. and Dresselhaus, M. S., "Effect of quantumwell structures on the thermoelectric figure of merit", Phys. Rev. B, Vol. 47 pp. 12727-12731, (1993). 